\title{
5-aminoimidazole-4-carboxamide ribonucleoside induces differentiation in a subset of primary acute myeloid leukemia blasts
}

Vilma Dembitz ${ }^{1,2}$, Hrvoje Lalic ${ }^{1,2}$, Ivan Kodvanj ${ }^{1,2}$, Barbara Tomic ${ }^{1,2}$, Josip Batinic ${ }^{3}$, Klara Dubravcic ${ }^{4}$, Drago Batinic ${ }^{2,4}$, Antonio Bedalov ${ }^{5}$ and Dora Visnjic ${ }^{1,2^{*}}$

\begin{abstract}
Background: All-trans retinoic acid (ATRA)-based treatment of acute promyelocytic leukemia (APL) is the most successful pharmacological treatment of acute myeloid leukemia (AML). Recent development of inhibitors of mutated isocitrate dehydrogenase and dihydroorotate dehydrogenase $(\mathrm{DHODH})$ has revived interest in differentiation therapy of non-APL AML. Our previous studies demonstrated that 5-aminoimidazole-4-carboxamide ribonucleoside (AICAr) induced differentiation of monocytic cell lines by activating the ATR/Chk1 via pyrimidine depletion. In the present study, the effects of AICAr on the viability and differentiation of primary AML blasts isolated from bone marrow of patients with non-APL AML were tested and compared with the effects of DHODH inhibitor brequinar and ATRA.
\end{abstract}

Methods: Bone marrow samples were obtained from 35 patients and leukemia blasts were cultured ex vivo. The cell viability was assessed by MTT assay and AML cell differentiation was determined by flow cytometry and morphological analyses. RNA sequencing and partial data analysis were conducted using ClusterProfiler package. Statistical analysis was performed using GraphPad Prism 6.0.

Results: AICAr is capable of triggering differentiation in samples of bone marrow blasts cultured ex vivo that were resistant to ATRA. AICAr-induced differentiation correlates with proliferation and sensitivity to DHODH inhibition. RNA-seq data obtained in primary AML blasts confirmed that AICAr treatment induced downregulation of pyrimidine metabolism pathways together with an upregulation of gene set involved in hematopoietic cell lineage.

Conclusion: AICAr induces differentiation in a subset of primary non-APL AML blasts, and these effects correlate with sensitivity to a well-known, potent DHODH inhibitor.

Keywords: AICAr, Acute myeloid leukemia, Differentiation, Brequinar, ATRA

\footnotetext{
* Correspondence: visnjic@mef.hr

${ }^{1}$ Croatian Institute for Brain Research, University of Zagreb School of

Medicine, 10000 Zagreb, Croatia

${ }^{2}$ Department of Physiology, University of Zagreb School of Medicine, Zagreb,

Croatia

Full list of author information is available at the end of the article
}

(c) The Author(s). 2020 Open Access This article is licensed under a Creative Commons Attribution 4.0 International License, which permits use, sharing, adaptation, distribution and reproduction in any medium or format, as long as you give appropriate credit to the original author(s) and the source, provide a link to the Creative Commons licence, and indicate if changes were made. The images or other third party material in this article are included in the article's Creative Commons licence, unless indicated otherwise in a credit line to the material. If material is not included in the article's Creative Commons licence and your intended use is not permitted by statutory regulation or exceeds the permitted use, you will need to obtain permission directly from the copyright holder. To view a copy of this licence, visit http://creativecommons.org/licenses/by/4.0/ The Creative Commons Public Domain Dedication waiver (http://creativecommons.org/publicdomain/zero/1.0/) applies to the data made available in this article, unless otherwise stated in a credit line to the data. 


\section{Background}

Acute myeloid leukemia (AML) is a severe hematological disorder that is characterized by the clonal expansion of myeloid blasts arrested at different stages of differentiation. AML is the most common acute type of leukemia in adults, with a median age at diagnosis of 68 years, the high risk of relapse and high mortality rate. The standard cytotoxic therapy consists of 7 days of cytarabine combined with 3 days of an anthracycline as a remission induction therapy, and several courses of high dose cytarabine or allogeneic hematopoietic stem cell transplantation as a consolidation therapy. With this therapy, long-term survival for patients with AML is achieved in only 35-45\% of those younger than 60 years of age and $10-15 \%$ of those aged 60 years and older. In elderly patients for whom intensive therapy is not appropriate, treatment remains unsatisfactory and includes low-dose cytarabine or the hypomethylating agents with supportive care. Since 2017, several new drugs have been approved for the treatment of AML, including FLT3 inhibitors, the CD33-directed antibodies and inhibitors of mutated isocitrate dehydrogenase (IDH) [1, 2]. However, the most successful pharmacological treatment of AML is still alltrans retinoic acid (ATRA)-based differentiation therapy of acute promyelocytic leukemia (APL), a particular subtype of AML defined by the PML-RARA rearrangement. Once fatal disease, APL has been successfully treated for the last three decades with ATRA and chemotherapy with complete remission (CR) rates of 90 to $95 \%$, and 85 to $90 \%$ rates of long-term survival. The clinical outcome of the disease has been further improved by the introduction of arsenic trioxide (ATO) into the treatment of refractory or relapsed APL, or as a first-line treatment in combination with ATRA [3].

The proposed mechanism of ATRA action involves binding of ATRA to the fusion protein promyelocytic leukemia $(\mathrm{PML}) /$ retinoic acid receptor $\alpha(\mathrm{RAR} \alpha)$ that is encoded by a translocation involving $P M L$ gene on chromosome 15 and $R A R A$ gene on chromosome 17, and which is assumed to act as a co-repressor. Therefore, ATRA-based therapy is reserved for cells having PML-RARA fusion product, which account for only 10$15 \%$ of AML, and all other non-APL AML are treated with cytotoxic drugs. However, it should be noted that differentiative properties of ATRA were first described in HL-60 cell line [4] established from peripheral blood of a patient suffering from AML-M2 which actually lack the PML-RARA rearrangement, and several studies showed that ATRA may drive leukemic cells efficiently into differentiation and/or apoptosis in a subset of nonAPL AML patients [5]. Recent findings that inhibitors of mutated isocitrate dehydrogenase (IDH) are associated with clinical and morphological signs of myeloid differentiation revived the interest in differentiation therapy of non-APL AML. Mutations in IDH genes are present in $18-22 \%$ of AML and lead to accumulation of the oncometabolite R-2-hydroxyglutarate (2-HG), which ultimately creates epigenetic alterations and differentiation arrest of AML blasts. IDH inhibitors restore normal enzyme activity, reduce 2-HG and induce differentiation $[6,7]$ being well-tolerated even in elderly patients with multiple comorbidities [8]. The IDH2 inhibitor enasidenib and the IDH1 inhibitor ivosidenib received FDA approval in 2017 and 2018 for selected patients with $I D H 2$ - and IDH1-mutated relapsed and refractory $(\mathrm{R} / \mathrm{R}) \mathrm{AML}$, respectively.

The success of IDH inhibitors invigorated interest in the role of metabolism in differentiation so that several new enzymes have been reported as possible targets for differentiation of AML, including methylenetetrahydrofolate dehydrogenase-cyclohydrolase 2 (MTHFD2) [9], dihydroorotate dehydrogenase (DHODH) [10] and lysine-specific demethylases [11]. Our previous studies demonstrated that 5aminoimidazole-4-carboxamide ribonucleoside (AICAr), a widely used agonist of AMP-activated kinase (AMPK), induces differentiation of monocytic leukemia cell lines in an AMPK-independent manner [12]. Ribotide AICAR was shown to interfere with pyrimidine synthesis by inhibiting UMP-synthase, which is a step downstream of DHODH; AICAr and the DHODH inhibitor brequinar had similar effects on differentiation and S-phase arrest, and the effects of both drugs depended on the activation of the DNA damage response ATR/Chk1 pathway [13]. In last couple of years, multiple agents have been identified as potent inhibitors of DHODH that trigger some differentiation of AML cell lines [14-19], patient AML cells ex vivo [19] or patient-derived xenografts in vivo $[18,19]$, and four clinical trials are underway in order to establish their safety and efficiency in AML patients [20].

The present study is a continuation of our previous work demonstrating the mechanism of AICAr-mediated differentiation $[12,13]$ that is undertaken in order to determine whether data obtained in AML cell lines could be translated to primary AML samples. Results of the study show that AICAr is capable of triggering differentiation in samples of bone marrow blasts cultured ex vivo that were resistant to ATRA. AICAr-induced differentiation correlates with proliferation and sensitivity to DHODH inhibition.

\section{Methods \\ Reagents}

Reagents used are listed in Supplementary Table 1. 


\section{Cell culture}

Bone marrow samples were obtained from 35 patients with non-APL AML at the time of diagnosis. None of the patients have received any treatment before specimens were collected. All patients provided written informed consent in accordance with the Declaration of Helsinki and the study was approved by the Institutional Review Board of the University of Zagreb School of Medicine (380-59-10,106-17-100/94) and University Hospital Center Zagreb (02/21 AG). In routine diagnostic procedures, all patients were tested for cytogenetic abnormalities and FLT3 mutations. Patients with normal karyotypes were additionally tested for NPM1 mutations. Depending on the FAB subtype, further analyses of BCR-ABL, AML1-ETO, PML-RARA, CBFB/MYH11 and MLL-AF4/AF9 were performed.

Mononuclear cells from bone marrow aspirates were isolated by density gradient centrifugation with NycoPrep $^{\mathrm{Tw}} 1.077$, further purified by overnight adherence to plastic and cryopreserved in liquid nitrogen. Thawed cells were cultured at starting concentration $0.4 \times 10^{6}$ / $\mathrm{mL}$ in RPMI 1640 containing 10\% FBS, 2 mM L-glutamine, $50 \mathrm{U} / \mathrm{mL}$ penicillin and $50 \mu \mathrm{g} / \mathrm{mL}$ streptomycin supplemented with $50 \mathrm{ng} / \mathrm{mL}$ interleukin-3 (IL-3), interleukin-6 (IL-6), FLT3 ligand (FLT3L) and stem cell factor (SCF) as previously described [21] and treated with agents tested. Only 16 samples with cell concentration higher than $0.2 \times 10^{6} / \mathrm{mL}$ in control conditions after $72 \mathrm{~h}$ culture were considered viable and included in further analysis. AML patients' characteristics are presented in Supplementary Table 2.

U937 cell line (ECACC number 85011440) was purchased from the European Collection of Authenticated Cell Cultures (Porton, Salisbury, UK). Cell line was expanded and frozen at early passages and used for the experiments within 10 weeks after being thawed from frozen stocks. The cells were maintained in RPMI 1640 medium containing $10 \% \mathrm{FBS}, 2 \mathrm{mML}$-glutamine, $50 \mathrm{U} /$ $\mathrm{mL}$ penicillin and $50 \mu \mathrm{g} / \mathrm{mL}$ streptomycin at $37^{\circ} \mathrm{C}$ in a humidified atmosphere containing $5 \% \mathrm{CO}_{2}$. For the experiments, cells were harvested, resuspended in fresh medium, seeded at a concentration of $0.2 \times 10^{6} / \mathrm{mL}$ in 6 well plates and treated with or without $0.2 \mathrm{mM}$ AICAr for $24 \mathrm{~h}$.

\section{Cell viability}

The total cell number and viability were assessed by counting on hemocytometer using trypan blue exclusion and by 3-(4,5-dimethylthiazol-2-yl)-2,5-diphenyltetrazolium bromide (MTT) assay. For MTT assay, cells were grown in triplicate with agents tested in 96-well plates for $96 \mathrm{~h}$. After addition of MTT solution, cells were incubated for additional $3 \mathrm{~h}$ at $37^{\circ} \mathrm{C}$, the insoluble formazan crystals obtained by centrifugation were dissolved in
DMSO and the absorbance was read at $595 \mathrm{~nm}$ with the reference wavelength of $655 \mathrm{~nm}$ using BioRad 680XR plate reader (Hercules, CA, USA).

\section{Flow cytometry}

Cultured cells were washed, aliquoted in three tubes and incubated with 8\% Fc Receptor Blocking solution for 10 min. Cells in first tube were stained with anti-CD11bFITC, anti-CD45-PerCP and anti-CD34-APC, in second tube with anti-CD64-FITC and in third tube with their respective isotypic controls for additional $20 \mathrm{~min}$ at room temperature in the dark. When used to test for cell viability of gated population, 7-AAD was added to tube containing anti-CD64 FITC for the last 2 min of incubation. Flow cytometry analyses were performed using FACS Calibur system (Becton Dickinson Immunocytometry Systems, San Jose, CA, USA). Cells were gated based upon forward and side scatter patterns and a total of 10,000 events were collected for each marker from this gated area. The obtained data were analyzed using CellQuest software and FlowJo v.10 platform. Mean fluorescence intensity (MFI) of the sample was calculated by subtracting MFI levels of isotypic controls from MFI levels of the cells stained with CD-specific antibodies. Percentage of positive cells was determined by measuring the fluorescence shift of distinct cluster of leukemic events.

For cell cycle analysis, cells were stained with propidium iodide (PI) solution $(50 \mu \mathrm{g} / \mathrm{mL}$ of PI, $10 \mathrm{mM}$ Tris, $\mathrm{pH}$ 8.0, $10 \mathrm{mM} \mathrm{NaCl}, 10 \mu \mathrm{g} / \mathrm{mL}$ RNase A, $0.1 \%$ Igepal) at $+4{ }^{\circ} \mathrm{C}$ for $20 \mathrm{~min}$. DNA analysis was performed by collecting 10,000 events for each sample gated in order to eliminate aggregates and cell debris using FACSCalibur System, while representative histograms were created by using FlowJo (LLC, Ashland, OR, USA).

\section{Microscopic analysis}

Samples were cytospun on microscopic slides using a StatSpin Cytofuge 2 (1000 rpm, $2 \mathrm{~min}$ ) and left to dry overnight. Slides were stained with May-Grünwald stain (50\% working solution, $5 \mathrm{~min}$ ) and, subsequently, Giemsa stain (10\% working solution, $20 \mathrm{~min})$. Morphology was examined using AxioVert 200 microscope and images were photographed using Axiocam MRc 5 camera and ZEN software, blue edition (Carl Zeiss AG, Oberkochen Germany).

\section{Automated image analysis}

Microscopic images were analyzed using CellProfiler and CellProfiler Analyst software as previously described [22]. Briefly, raw color images were split to red, green and blue channels, intensity was rescaled and segmentation was performed on green channel identifying nuclei as primary, whole cells as secondary and cytoplasm as 
tertiary objects. Cytological profile was generated for every cell using "Measure Object Size Shape" module in CellProfiler. Subsequent analysis was performed using CellProfiler Analyst in order to classify cells into three phenotypic categories: macrophage-like, negative and dead cells. A Random Forrest Classifier model used for this purpose was trained by manually classifying 812 randomly fetched objects (cells) and was evaluated by constructing confusion matrix. CellProfiler pipelines, CellProfiler classifier model and training set are available on request.

\section{RNA-sequencing and data analysis}

AML sample Pt 14 and U937 cells were cultured as described above and treated with 0.4 and $0.2 \mathrm{mM}$ AICAr, respectively, for $24 \mathrm{~h}$. RNA isolation, sample quality assessment, RNA library preparation, sequencing and partial data analysis (raw data quality control, trimming, mapping, aligning and calculating gene hit counts) were conducted at GENEWIZ, Inc. (South Plainfield, NJ, USA). The RNA samples were quantified using Qubit 2.0 Fluorometer (Life Technologies, Carlsbad, CA, USA) and RNA integrity was checked with Agilent TapeStation (Agilent Technologies, Palo Alto, CA, USA). NEBNext Ultra RNA Library Prep Kit for Illumina (NEB, Ipswich, MA, USA) was used for preparation of cDNA libraries and sequencing was performed on the Illumina HiSeq 4000 or equivalent instrument in High Output Mode $(2 \times 150$ Paired End configuration).

After investigating the quality of the raw data, sequence reads were trimmed using Trimmomatic v.0.36 and mapped to the reference genome Homo sapiens GRCh38 using the STAR aligner v.2.5.2b. Unique gene hit counts were calculated from BAM files by using feature Counts from the Subread package v.1.5.2.

Downstream differential expression analysis and functional analyses were performed entirely in R. To normalize counts and perform differential expression analysis DESeq2 was used (v. 1.24.0) with $p$-value threshold $<0.05$ and $\log 2$ fold change threshold $>1$. ClusterProfiler package [23] was used to further analyze differentially expressed genes by gene set enrichment analysis (GSEA) on KEGG gene sets. The GSEA algorithm was run using log fold change values as the genelevel statistics, 100,000 random permutations.

Publicly available datasets TCGA AML [24], GSE15434 [25] and OHSU BeatAML [26] were used for expression analysis of uridine monophosphate synthase (UMPS) and DHODH in AML patients. Data was accessed through Bloodspot [27] and cBioPortal [28]. Difference in gene expression levels between different groups were assessed using ANOVA with post hoc Tukey test and $p$-values $<0.05$ were considered statistically significant. Correlation in gene expression was assessed using both Pearson's and Spearman's correlation coefficients and results in which both $p<0.05$ and $\mathrm{q}<0.05$ were considered statistically significant.

\section{Statistical analysis}

Apart from sequencing data, all other data are presented as mean \pm standard error of the mean (S.E.M). Difference between groups was determined using ANOVA with post-hoc Dunnet test for comparison of groups to control group and $p$-values $<0.05$ were considered statistically significant. Statistical analysis was performed using GraphPad Prism 6.0.

\section{Results}

\section{AICAr decreases cell viability in primary AML samples}

To screen first for the possible antiproliferative effects of AICAr in primary AML samples, an initial set of bone marrow samples from 35 patients suffering from nonAPL AML was collected. Mononuclear cells were isolated from bone marrow samples by density gradient centrifugation, allowed to adhere overnight on plastic, and the non-adherent cells were grown in vitro in RPMI medium with the addition of IL-3, IL-6, SCF and FLT3L. Without any drugs added, 16 samples had more than $0.2 \times 10^{6}$ of viable cells per $\mathrm{mL}$ after $72 \mathrm{~h}$ of incubation (Fig. 1a). These samples were included in further analyses, and patient characteristics are presented in Supplementary Table 2.

The results of MTT assay showed that AICAr significantly reduced the viability of patient samples in a concentration-dependent manner (Fig. 1b). Knowing that AICAr exerts metabolic effects that may affect the results of MTT assay [29], the reduction in cell viability was confirmed by simple counting of trypan bluenegative cells on the hemocytometer (Fig. 1c). These results demonstrate that AICAr decreases cell viability in primary AML samples.

\section{AICAr and brequinar induce differentiation in a subset of primary AML samples that are resistant to ATRA}

AICAr, brequinar and ATRA were then tested for the capability to induce differentiation in sixteen viable samples. As shown in Fig. 1a, only five of these samples exhibited proliferation in the absence of drugs, as judged by having the number of viable cells above plating density (more than $0.4 \times 10^{6}$ ) after $72 \mathrm{~h}$. Two out of these proliferating samples exhibited upregulation of the differentiation marker CD11b upon AICAr treatment; FAB-M2 and FAB-M4 (Pt 14 and Pt 07).

In one patient sample ( $\mathrm{Pt}$ 14: FAB-M2, normal karyotype, FLT3wt NPM1wt, primary refractory), the addition of AICAr in concentrations that reduced the number of leukemia cells resulted in a pronounced increase in the expression of markers CD11b and 
a)

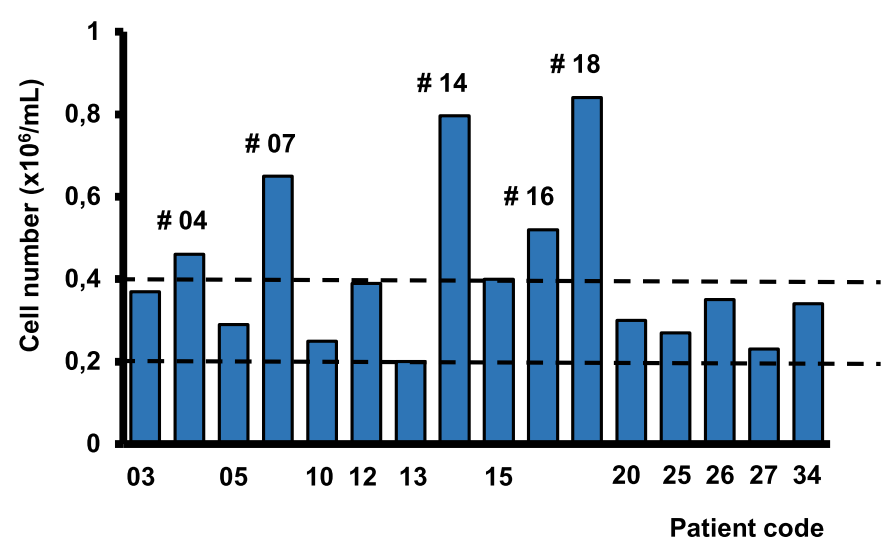

b)

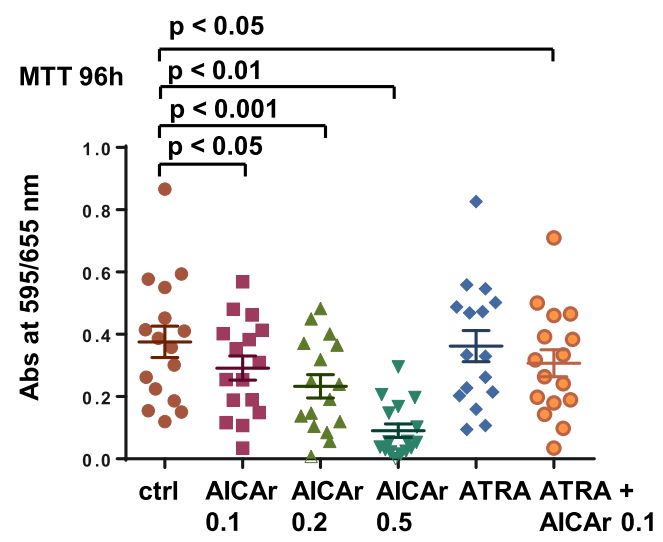

c)

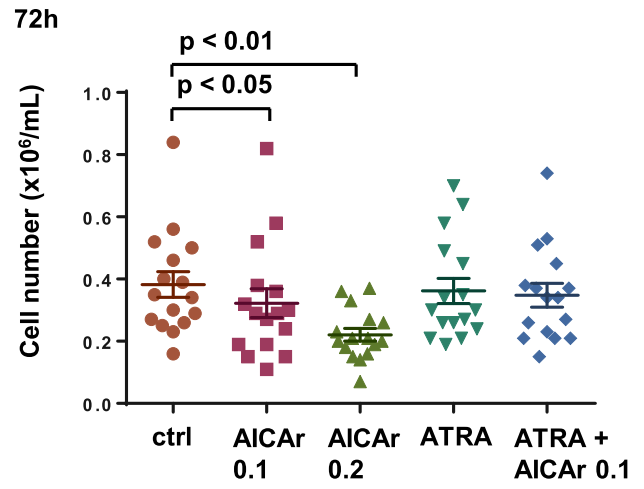

Fig. 1 AICAr decreases cell viability in primary AML samples. Bone marrow samples of $16 \mathrm{AML}$ patients were seeded at concentration $0.4 \times 10^{6}$ \% $\mathrm{mL}$ in medium supplemented with $50 \mathrm{ng} / \mathrm{mL} \mathrm{IL-3,} \mathrm{IL-6,} \mathrm{SCF} \mathrm{and} \mathrm{FLT3L} \mathrm{and} \mathrm{incubated} \mathrm{with} \mathrm{agents} \mathrm{tested.} \mathrm{a)} \mathrm{The} \mathrm{number} \mathrm{of} \mathrm{viable} \mathrm{control} \mathrm{cells} \mathrm{in}$ patient samples after $72 \mathrm{~h}$ as determined by trypan blue exclusion. b) Cells were incubated in triplicates with AICAr (0.1-0.5 mM), ATRA (1 $\mu \mathrm{M})$, and combination of $\operatorname{AlCAr}(0.1 \mathrm{mM})$ and ATRA $(1 \mu \mathrm{M})$ in 96-well plates and MTT assay was performed after $96 \mathrm{~h}$. The mean value of MTT assay is presented for every patient. Results are mean \pm S.E.M, $N=16$ c) Primary samples were incubated with agents tested in 12 -well plates and the number of viable cells was determined after $72 \mathrm{~h}$ by trypan blue exclusion. Results are mean \pm S.E.M, $N=16$. Statistical significance was determined using one-way ANOVA and post-hoc Dunnett test

CD64, as well as an increase in percentage of CD $45^{\text {high }}$ cells, suggesting the differentiation of blasts to more mature forms (Fig. 2a \& b). Immunophenotypic changes in AICAr-treated cells were accompanied by accumulation of cells that morphologically resembled macrophages as they show an increase in the cell size, the vacuolization of the cytoplasm and a decrease in nucleocytoplasmic ratio. The similar effects were observed in cells treated with brequinar, and no significant 


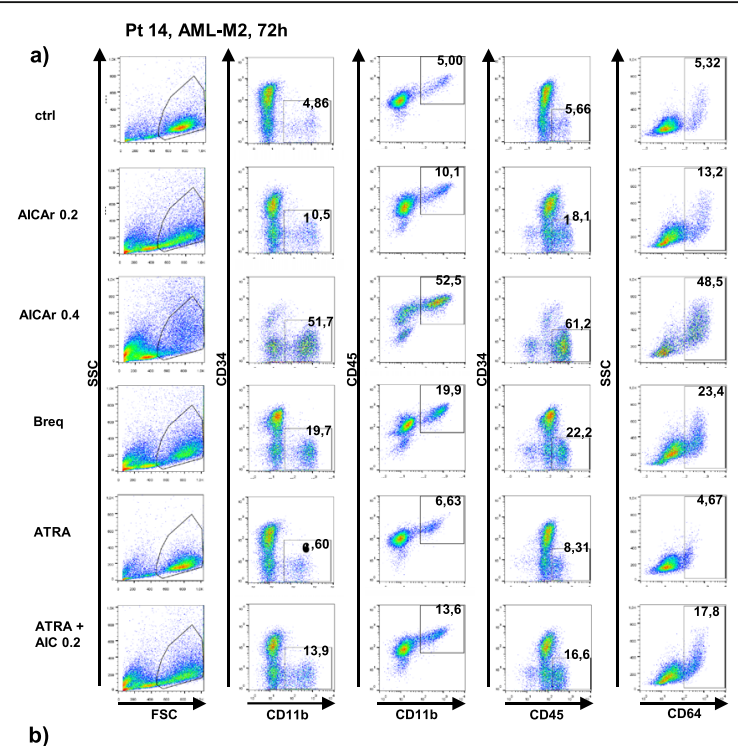

\section{c)}

$100 x \quad$ ctrl
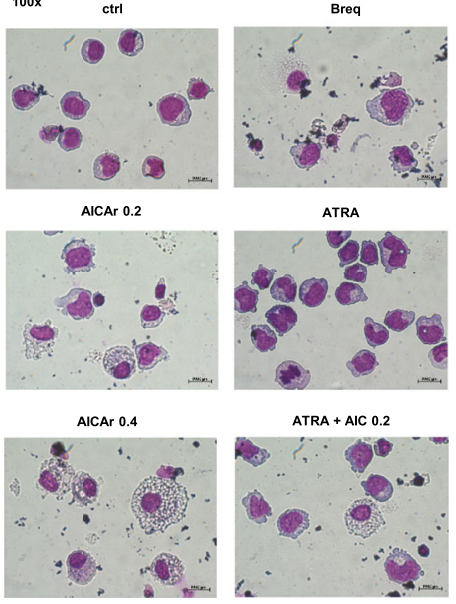

ATRA + AIC 0.2

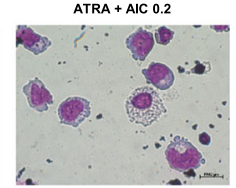

d)
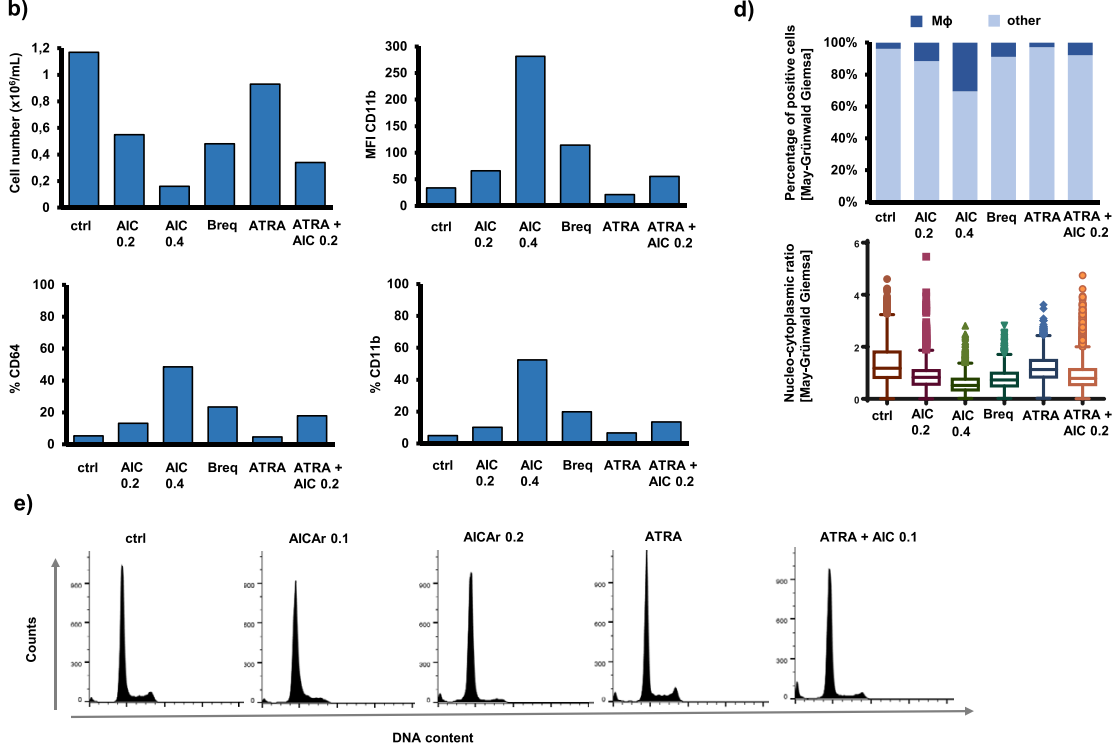

Fig. 2 AICAr induces differentiation and accumulation of macrophage-like cells in a primary sample from AML-M2 patient (normal karyotype, FLT3wt NPM1wt, primary refractory). Bone marrow sample (Pt 14) was seeded at concentration $0.4 \times 10^{6} / \mathrm{mL}$ in medium supplemented with 50 $\mathrm{ng} / \mathrm{mL} \mathrm{IL}-3, \mathrm{IL}-6, \mathrm{SCF}$ and FLT3L and incubated with AICAr (0.2 and $0.4 \mathrm{mM})$, ATRA $(1 \mu \mathrm{M})$, combination of AICAr $(0.2 \mathrm{mM})$ and ATRA $(1 \mu \mathrm{M})$, or brequinar ( $500 \mathrm{nM}$ ) for $72 \mathrm{~h}$. a) Flow cytometric analysis of $\mathrm{CD} 11 \mathrm{~b}^{+} \mathrm{CD} 34, \mathrm{CD} 11 \mathrm{~b}^{+} \mathrm{CD} 45^{+}, \mathrm{CD} 45^{\text {high }} \mathrm{CD} 34^{-}$and $\mathrm{CD} 64^{+}$populations. Percentage of cells in population of interest is indicated in respective gates. b) The number of viable cells was determined by trypan blue exclusion. Mean fluorescence intensity (MFI) of CD11b and percentage of CD64 and CD11b positive cells were determined by flow cytometry. c) May-GrünwaldGiemsa stained cytospin preparations (100x magnification). d) Proportion of macrophage-like cells in samples and nucleo-cytoplasmic ratio of cells determined using automated image analysis of at least 1000 cells per representative cytospin preparation (10x magnification). e) Cell cycle analysis of propidium-labeled cells. Results are representatives of three independent experiments

differentiative effects were seen in cells treated with ATRA (Fig. 2c \& d). In addition, cell cycle analysis of propidiumlabeled cells revealed a decrease in $\mathrm{G}_{2} / \mathrm{M}$ phase of AICArtreated cells and inhibition of progression through the $\mathrm{S}$ phase (Fig. 2e).

In a sample of more mature myelomonocytic cells (Pt 07: FAB-M4, normal karyotype, FLT3-ITD, NPM1mut), the basal level of $\mathrm{CD} 11 \mathrm{~b}$ positive cells was higher, but further increased in the presence of both AICAr and brequinar (Fig. 3a). Moreover, the increase in the expression of CD11b was associated with an increase in CD64 expression, a decrease in cell number (Fig. $3 \mathrm{~b}$ ) and morphological changes associated with differentiation into macrophage-like cells (Fig. 3c). Again, no increase in the level of CD11b was seen in cells treated with ATRA.

We concluded that AICAr-induced differentiation in a subset of primary non-APL AML blasts had no correlation with either FAB classification, cytogenetics or mutational 


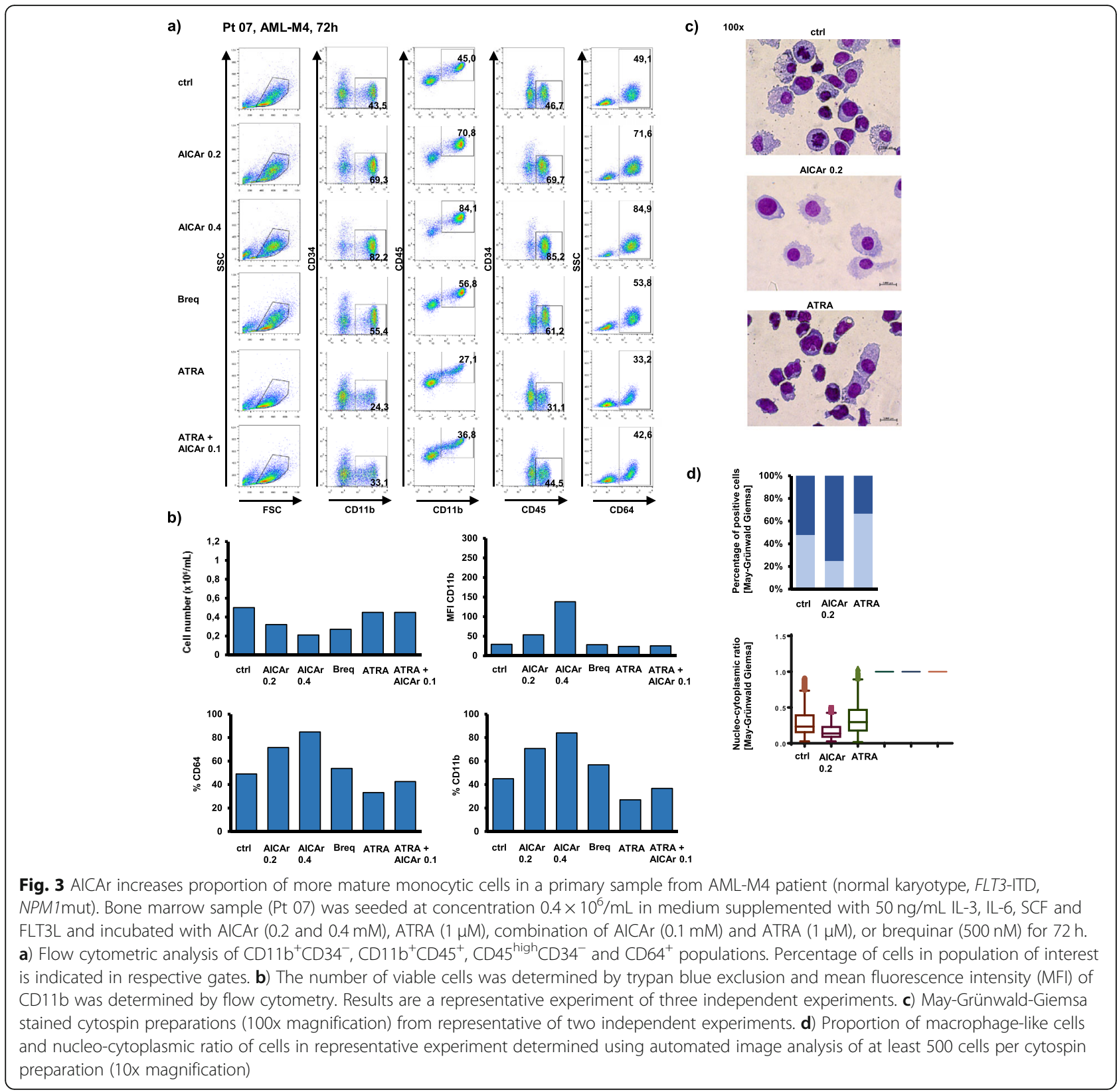

status of FLT3 or NPM1. AICAr-sensitive primary AML samples differentiated in the presence of brequinar, but were resistant to differentiative effects of ATRA. To further test if there is any connection between the expression of UMPS and DHODH, which are presumed molecular targets of AICAr and brequinar, and patients cytogenetic and genetic signature, we performed the analysis on publicly available datasets. Expression analysis of DHODH and UMPS in TCGA and GSE15434 datasets showed no significant differences in the expression of these genes in AML patients depending on the cytogenetics or mutational status in CEBPA, FLT3 or NPM1 (Supplementary Fig. 1A), and the expression of both $D H O D H$ and $U M P S$ genes was inversely correlated with the expression of ITGAM (CD11b) in TCGA and OHSU BeatAML datasets (Supplementary Fig. 1B).

\section{AICAr treatment changes the expression of genes involved in regulation of cell cycle and pyrimidine metabolism in responsive AML patient}

To elucidate the effects of AICAr on gene expression in primary AML cells, we performed RNA sequencing of 
control and AICAr-treated cells isolated from bone marrow of AML patient (Pt 14) in which AICAr induced the strongest differentiative response. RNA was isolated from cells that were cultured in triplicates and treated with or without $0.4 \mathrm{mM}$ AICAr for $24 \mathrm{~h}$. As shown in Fig. 4a, genes associated with monocyte/macrophage differentiation (SPP1, THBD, CCL7...), and those associated with neutrophil function (MPO, PRTN3...) were among top significant genes regulated by AICAr. Furthermore, hierarchical clustering of top 50 significant genes revealed a large proportion of genes associated with DNA replication and cell cycle (PHLDA1, MCM, CLSPN, CHEK1, MYBL2, ZWINT, CDC6, CDC25A) as well as several genes related to purine and pyrimidine metabolism (TK1, TYMS, DHFR) (Fig. 4b). In addition, gene set enrichment analysis (GSEA) on KEGG database was performed and complete results are presented in Supplementary Table 3. As shown in Fig. 4c, GSEA on KEGG a)

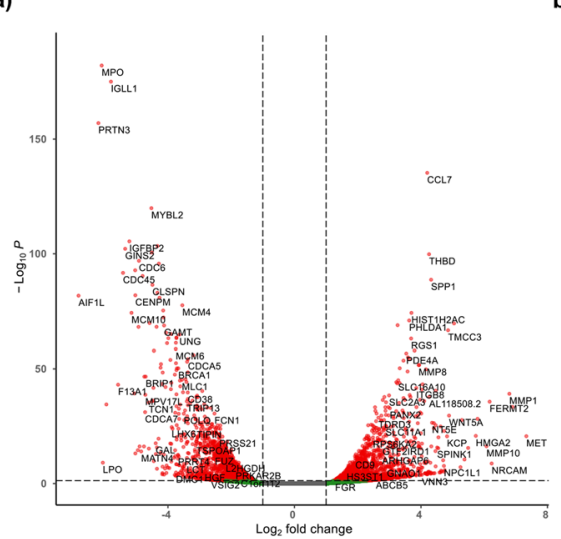

c)

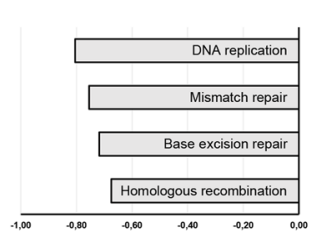

b)

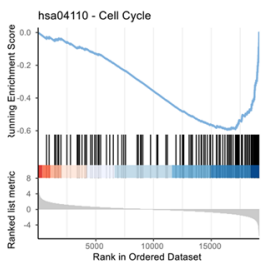

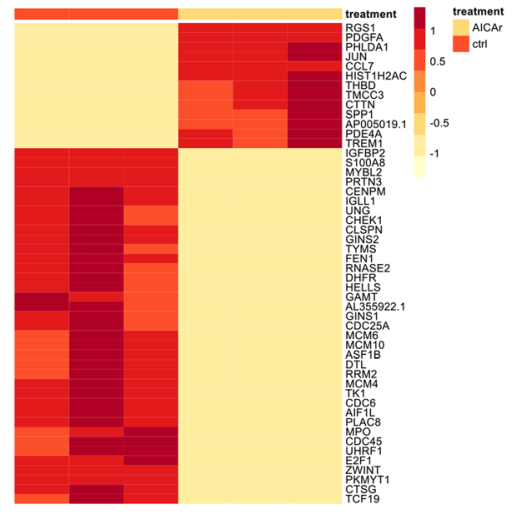
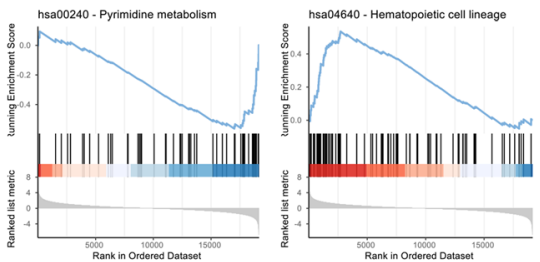

U937, $24 \mathrm{~h}$

d)

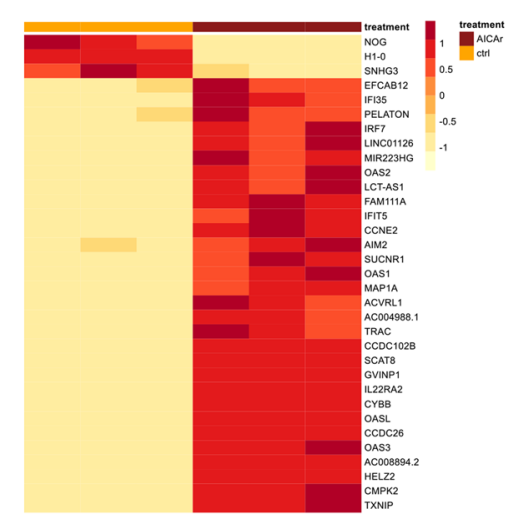

e)

\begin{tabular}{|l|l|l|}
\hline Gene & $\begin{array}{l}\text { Log2 Fold change } \\
\text { Pt14 }\end{array}$ & $\begin{array}{l}\text { Log2 Fold change } \\
\text { U937 }\end{array}$ \\
\hline CD34 & -1.70 & -0.10 \\
\hline KIT/CD117) & -1.49 & -0.26 \\
\hline MPO & -6.13 & 0.02 \\
\hline LYZ & -1.76 & 0.63 \\
\hline CD14 & -1.06 & 0.00 \\
\hline IL1R1 & 2.96 & -0.03 \\
\hline IL1R2 & 1.92 & -0.03 \\
\hline IL1A & 2.11 & 0.14 \\
\hline IL1B & 2.47 & 1.20 \\
\hline FCGR1A(CD64) & 1.14 & 0.52 \\
\hline FCGR2A & 2.42 & 0.04 \\
\hline ITGAM (CD11B) & 1.64 & 0.67 \\
\hline CSF1R & 1.53 & 0.30 \\
\hline SOCS3 & 1.63 & 0.01 \\
\hline CD58 & 1.37 & 0.26 \\
\hline CD163 & 1.26 & 0.03 \\
\hline CCR2 & -3.23 & 0.27 \\
\hline CCR1 & 1.89 & 0.99 \\
\hline CCR7 & 1.66 & 0.06 \\
\hline & & \\
\hline
\end{tabular}

Fig. 4 Differential gene expression in primary sample from AML-M2 patient (normal karyotype, FLT3wt NPM1wt, primary refractory) and U937 cells in response to AICAr. Bone marrow sample from the AML patient (Pt 14) in which AICAr induced differentiation in vitro and U937 cells were seeded in triplicates and treated with AICAr for $24 \mathrm{~h}$. Differential gene expression was determined using RNA sequencing and DESeq2 analysis was performed. a) Volcano plot. The red dots: $|\log 2 \mathrm{FC}| \geq 1$, p.adjusted < 0.05; the green dots: $|\log 2 \mathrm{FC}| \geq 1$, p.adjusted $>0.05$; the black dots: $\mid \log 2$ $\mathrm{FCl} \leq 1$, p.adjusted $>0.05$. b) Heatmap from hierarchical clustering of top 50 significant genes ranked by p.adjusted value between untreated and AICAr-treated primary samples. c) Gene Set Enrichment Analysis (GSEA) for KEGG pathways: Top 4 most enriched pathways and GSEA plots (score curves) for pathways Cell Cycle, Pyrimidine Metabolism and Hematopoietic Cell Lineage. p.adjusted < 0.01 . d) Heatmap from hierarchical clustering of top 30 significant genes ranked by p.adjusted value between control and AlCAr-treated U937 cells. e) Log2Fold changes in the expression of genes associated with immature cells, monocytes and macrophages in primary sample and U937 cells 
pathways confirmed that AICAr treatment induced a strong downregulation of cell cycle and pyrimidine metabolism pathways together with a strong upregulation of gene set involved in hematopoietic cell lineage.

To compare the transcriptional profile of AICArtreated primary AML cells with the effects of AICAr in monocytic U937 cell line in which AICAr-mediated differentiation was first observed [12], RNA sequencing was performed in control and AICAr-treated U937 cells (Fig. 4d). As shown in Fig. 4e, an increase in the expression of genes associated with macrophage differentiation (e.g. CSF1R, SOCS3, CD163, CCR1, CCR7...) followed by a decrease in the expression of markers of immature cells (CD34 and KIT/CD117) or monocytes (CCR2) was observed in both primary AML cells and U937 cell line. In addition, list of common gene expression changes (Supplemental Table 4) included genes associated with cytokines, chemokines and their receptors (IL1B, IL1RN, CCL2, CCL3, CCR1), metabolism (MAN1B1-AS1, IPP, $P T G S 2, A B H D 4 \ldots)$ and cell cycle progression, DNA repair and apoptosis (CDKN1A, PPP1R10, PANO1...).

We conclude that RNA-seq data obtained in primary AML blasts treated with AICAr are in agreement with our previous data showing that AICAr-induced differentiation of AML cell lines depended on cell cycle arrest through pyrimidine depletion [13].

\section{Discussion}

AICA-ribonucleoside (AICAr) is an exogenous substance which, after entering cells, becomes phosphorylated into AICA-ribonucleotide (AICAR or ZMP), and AICAR is an evolutionary conserved cellular intermediate in de novo purine synthesis that accumulates in Lesch-Nyhan syndrome [30]. Although both AICAr and metformin have been widely used as AMPK agonists in studies related mostly to insulin signaling and metabolism, there are many recent studies showing that some of their effects on cell cycle, growth and metabolism are actually AMPK-independent [31, 32]. Because of structural similarities with adenosine, AICAr was first used to prevent cardiovascular complications in patients undergoing coronary artery bypass surgery [33], but then became popular as an "exercise in a pill" as the study in mice revealed that AICAr increased endurance and mimicked the effects of aerobic exercise [34]. The most recent interest in AICAr was gained by studies reporting antitumor effects of metformin and other AMPK agonists in cancer [35]. Cytotoxic effects of AICAr in vitro have been demonstrated in several hematological malignancies at concentrations that are well tolerated when achieved in plasma after intravenous injection [36-38], and results of clinical trial testing the effects in $\mathrm{B}$ cell chronic lymphocytic leukemia showed that AICAr had an acceptable safety profile and antileukemic activity in patients with poor prognosis. The pharmacokinetic analysis performed during that clinical trial demonstrated that at the maximum tolerated dose of $210 \mathrm{mg} / \mathrm{kg}$, the plasma concentration of AICAr and its metabolite ZMP corresponded to $0.9 \mathrm{mM}$ [39]. The results of the present study show that lower concentrations of AICAr exert significant antiproliferative effects in primary AML samples.

The antiproliferative effects of AICAr in AML cell lines were previously proved to be AMPKindependent [12]. Both AICAr and metformin induced apoptosis, but only AICAr induced differentiation that depended on the inhibition of UMPsynthase and was completely overcame by addition of uridine [13]. AICAr shares the same mechanisms of action with well-known inhibitors of de novo pyrimidine synthesis that have been previously shown to induce uridine-dependent leukemia differentiation, such as DHODH inhibitors brequinar [10] and leflunomide [40], or UMP synthase inhibitor pyrazofurin [10]. In AML cell lines, low concentrations of AICAr and brequinar were shown to exert synergistic effects on both cell cycle arrest in S-phase and cellular differentiation, and the effects of both agents depended on the activation of DNA damage ATR/Chk1 signaling pathway [13]. In this study, we present evidence that both drugs can induce an increase in the expression of differentiation markers and morphological changes associated with maturation in samples isolated from patients with AML, and these effects do not correlate with either FAB classification or mutational status of NPM1 and FLT3-ITD. However, the effects of both AICAr and brequinar on differentiation of primary AML blasts required proliferation in conditions in vitro, and this would be expected from the proposed mechanism of AICAr- and brequinar-mediated differentiation that depends on the lack of pyrimidine synthesis and the cell cycle arrest.

There is a considerable interest toward the development of new inhibitors of DHODH as a potential option for differentiation therapy of AML. Brequinar was tested in clinical trials for solid tumors, but myeloid suppression and severe adverse reactions limited its clinical application. In spite of known toxicity, a currently ongoing phase $1 \mathrm{~b} / 2 \mathrm{a}$ clinical trial (NCT03760666) is trying to assess its safety and efficacy in patients with relapsed/refractory AML [20]. Leflunomide and teriflunomide are FDAapproved for rheumatoid arthritis and multiple sclerosis, respectively, but they have failed to receive FDA approval for cancer [41]. The novel DHODH inhibitor BAY 240234 is currently under phase 1 clinical trial (NCT03404726) for myeloid malignancies, and preclinical data showed differentiative 
effects in several AML cell lines, including THP-1, and three subcutaneous AML xenografts in vivo [18]. Another novel DHODH inhibitor, ASLAN003 is currently being evaluated in a phase $2 \mathrm{a}$ trial (NCT03451084) in AML and preclinical data on primary AML blasts grown under similar conditions as the ones used in our study revealed sensitivity in six out of fourteen AML samples [19]. In study by Zhou at al., sensitivity was defined as an increase $\geq 15 \%$ in any of markers CD11b, CD14, CD13 or CD33 [19], while our present study defines response as a significant increase in the level of CD11b. However, both studies agree that differentiation of AML in response to ASLAN003, AICAr or brequinar is independent from FAB subtype or mutational status of FLT3 or NPM1.

Results of our study show that sensitivity to AICAr correlates well with sensitivity to brequinar in primary AML samples. Although there is a potential mechanistic link in the activity of pyrimidine pathway and differentiation blockade in AML patients, our analysis of the expression of DHODH and UMPS further suggests that patients who could potentially benefit from the modulation of the activity of pyrimidine synthesis pathway are not easily identifiable using conventional diagnostic markers in AML. Our previous work provided the link between the defect in pyrimidine synthesis, cell cycle arrest, ATR/Chk1 pathway and cellular differentiation [13] and present data obtained by RNA sequencing, albeit limited to just one patient sample, point to the similar direction. In contrast, none of the samples that responded to inhibitors of pyrimidine synthesis differentiated in the presence of ATRA. These results are in accordance with our previous data demonstrating that the effects of AICAr on metabolism and cell cycle progression differ from the effects of ATRA and that these two drugs act via independent mechanisms [13]. Further investigations of the pleiotropic effects of pyrimidine inhibition will be required to fully understand the mechanism and to identify the subtype of non-APL AML patients that may benefit from this differentiation therapy, but it is possible that the mechanism of differentiation more closely resembles that of low doses of cytotoxic drugs, and involves pathways that regulates cell cycle, DNA damage response and apoptosis.

\section{Conclusions}

The results of our present study demonstrate that AICAr induces differentiation in a subset of non-APL AML blasts isolated from bone marrow of patients suffering from AML, and these effects do not correlate with FAB classification or mutational status of FLT3 or NPM1, but correlate with sensitivity to well-known, potent DHODH inhibitor.

\section{Supplementary information}

Supplementary information accompanies this paper at https://doi.org/10. 1186/s12885-020-07533-6.

\section{Additional file 1 Supplementary Table 1. Reagents and resources} used.

Additional file $\mathbf{2}$ Supplementary Table 2. Patient characteristics. Additional file $\mathbf{3}$ Supplementary Table 3. Results of GSEA analysis on KEGG gene sets.

Additional file 4 Supplementary Table 4. Common gene expression changes of control and AICAr-treated bone marrow sample (Pt14) and U937 cells in relation to their respective untreated controls (selected from the results of the differential gene expression analysis conducted by GENEWIZ)

Additional file $\mathbf{5}$ Supplementary Figure 1. Expression of $\mathrm{DHODH}$ and UMPS in AML patient samples. A) Publicly available datasets TCGA and GSE15434 (accessed through Bloodspot database) with a total of 413 patients were used to compare expression levels of DHODH and UMPS in primary AML samples in accordance to their cytogenetics and mutational status of CEBPA, FLT3 and NPM1. B) Gene co-expression analysis between DHODH or UMPS and ITGAM (CD11b) was performed on publicly available datasets TCGA and OHSU (accessed through cBioPortal database) which jointly comprise 567 samples.

\section{Abbreviations}

AICAr: 5-aminoimidazole-4-carboxamide ribonucleoside; AICAR: 5aminoimidazole-4-carboxamide ribotide (ZMP); AML: Acute myeloid leukemia; AML1-ETO: Acute myeloid leukemia 1- Eight-twenty-one oncoprotein; AMP: Adenosine monophosphate; AMPK: AMP-activated kinase; APL: Acute promyelocytic leukemia; ATO: Arsenic trioxide; ATR/Chk1: Ataxia telangiectasia and Rad3-related protein/Checkpoint kinase 1; ATRA: All-trans retinoic acid; $B C R-A B L$ : Breakpoint cluster region-Abelson murine leukemia viral oncogene homolog 1; CBFB/MYH11: Core-binding factor subunit beta/ Myosin heavy chain 11; CCL7: C-C motif chemokine ligand 7; CDC25A: Cell division cycle 25A; CDC6: Cell division cycle 6; CHEK1: Checkpoint kinase 1; CLSPN: Claspin; DHFR: Dihydrofolate reductase; $\mathrm{DHODH}$ : Dihydroorotate dehydrogenase; DMSO: Dimethyl sulfoxide; FLT3: FMS-related tyrosine kinase 3; GSEA: Gene set enrichment analysis; IDH: Isocitrate dehydrogenase; ITD: Internal tandem duplication; KEGG: Kyoto Encyclopedia of Genes and Genomes; MCM: Minichromosome maintenance complex; MLL-AF4/ AF9: Lysine methyltransferase 2A-AF4/FMR2 family member 1/MLLT3 super elongation complex subunit; MPO: Myeloperoxidase; MTHF

D2: Methylenetetrahydrofolate dehydrogenase-cyclohydrolase 2; MTT: 3-(4,5dimethylthiazol-2-yl)-2,5-diphenyltetrazolium bromide; MYBL2: MYB protooncogene like 2; NPM1: Nucleophosmin 1; PHLDA1: Pleckstrin homology like domain family A member 1; PML/RARa: Promyelocytic leukemia/Retinoic acid receptor a; PRTN3: Proteinase 3; SCF: Stem cell factor; SPP1: Secreted phosphoprotein 1; THBD: Thrombomodulin; TK1: Thymidine kinase 1; TYMS: Thymidylate synthetase; UMP: Uridine monophosphate; ZMP: 5aminoimidazole-4-carboxamide ribotide (AICAR); ZWINT: ZW10 interacting kinetochore protein; 2-HG: R-2-hydroxyglutarate; 7-AAD: 7-aminoactinomycin D

\section{Acknowledgements}

We thank Ms. Marijana Andrijašević for valuable technical help and support.

\section{Authors' contributions}

VD, HL, IK, BT, and DV performed the research and analyzed data, JB recruited patients and collected samples, VD and IK performed transcriptome analysis, KD, DB and AB analyzed data, VD, AB and DV wrote the paper. All authors revised and approved the submitted version.

\section{Funding}

This work has been funded by Croatian Science Foundation under the projects IP-2016-06-4581 and DOK-2018-01-9599 by the European Union through the ESF Operational Programme Efficient Human Resources 20142020 (to D.V.), supported by National Institute of Health Grant R01GM117446 to $A B$, and co-financed by the Scientific Centre of Excellence for Basic, Clinical and Translational Neuroscience (project "Experimental and clinical research of hypoxic-ischemic damage in perinatal and adult brain"; GA 
KK01.1.1.01.0007) funded by the European Union through the European Regional Development Fund).

\section{Availability of data and materials}

The datasets generated and analysed during the current study are available in the Array Express Archive repository, https://www.ebi.ac.uk/arrayexpress/ experiments/E-MTAB-9209/, https://www.ebi.ac.uk/arrayexpress/experiments/ E-MTAB-9537/

\section{Ethics approval and consent to participate}

All patients included in this study provided written informed consent in accordance with the Declaration of Helsinki and the study was approved by the Institutional Review Board of the University of Zagreb School of Medicine (380-59-10106-17-100/94) and University Hospital Center Zagreb (02/21 AG).

\section{Consent for publication}

Not applicable.

\section{Competing interests}

The authors declare that they have no competing interests with the contents of this article.

\section{Author details}

${ }^{1}$ Croatian Institute for Brain Research, University of Zagreb School of Medicine, 10000 Zagreb, Croatia. ${ }^{2}$ Department of Physiology, University of Zagreb School of Medicine, Zagreb, Croatia. ${ }^{3}$ Division of Hematology, Department of Internal Medicine, University Hospital Center Zagreb, Zagreb, Croatia. ${ }^{4}$ Department of Laboratory Immunology, University Hospital Center Zagreb, Zagreb, Croatia. ${ }^{5}$ Clinical Research Division, Fred Hutchinson Cancer Research Center, Seattle, WA, USA.

\section{Received: 2 July 2020 Accepted: 15 October 2020}

\section{Published online: 11 November 2020}

\section{References}

1. Short NJ, Rytting ME, Cortes JE. Acute myeloid leukaemia. Lancet. 2018; 392(10147):593-606. https://doi.org/10.1016/S0140-6736(18)31041-9.

2. Estey EH. Acute myeloid leukemia: 2019 update on risk-stratification and management. Am J Hematol. 2018;93(10):1267-91. https://doi.org/10.1002/ ajh.25214.

3. Sanz MA, Fenaux P, Tallman MS, Estey EH, Löwenberg B, Naoe T, et al. Management of acute promyelocytic leukemia: updated recommendations from an expert panel of the European LeukemiaNet. Blood. 2019;133(15): 1630-43. https://doi.org/10.1182/blood-2019-01-894980.

4. Breitman TR, Selonick SE, Collins SJ. Induction of differentiation of the human promyelocytic leukemia cell line $(\mathrm{HL}-60)$ by retinoic acid. Proc Natl Acad Sci. 1980 May 1;77(5):2936-40. https://doi.org/10.1073/pnas.77.5.2936.

5. van Gils N, Verhagen HJMP, Smit L. Reprogramming acute myeloid leukemia into sensitivity for retinoic-acid-driven differentiation. Exp Hematol. 2017;52:12-23. https://doi.org/10.1016/j.exphem.2017.04.007.

6. Stein EM, DiNardo CD, Pollyea DA, Fathi AT, Roboz GJ, Altman JK, et al. Enasidenib in mutant IDH2 relapsed or refractory acute myeloid leukemia. Blood. 2017;130(6):722-31. https://doi.org/10.1182/blood-2017-04-779405.

7. Amatangelo MD, Quek L, Shih A, Stein EM, Roshal M, David MD, et al. Enasidenib induces acute myeloid leukemia cell differentiation to promote clinical response. Blood. 2017;130(6):732-41. https://doi.org/10.1182/blood2017-04-779447.

8. Pollyea DA, Tallman MS, de Botton S, Kantarjian HM, Collins R, Stein AS, et al. Enasidenib, an inhibitor of mutant IDH2 proteins, induces durable remissions in older patients with newly diagnosed acute myeloid leukemia. Leukemia. 2019;33(11):2575-84. https://doi.org/10.1038/s41375-019-0472-2.

9. Pikman Y, Puissant A, Alexe G, Furman A, Chen LM, Frumm SM, et al. Targeting MTHFD2 in acute myeloid leukemia. J Exp Med. 2016;213(7):1285306. https://doi.org/10.1084/jem.20151574.

10. Sykes DB, Kfoury YS, Mercier FE, Wawer MJ, Law JM, Haynes MK, et al. Inhibition of dihydroorotate dehydrogenase overcomes differentiation blockade in acute myeloid leukemia. Cell. 2016;167(1):171-86. https://doi. org/10.1016/j.cell.2016.08.057.

11. Maes T, Mascaró C, Tirapu I, Estiarte A, Ciceri F, Lunardi S, et al. ORY-1001, a potent and selective covalent KDM1A inhibitor, for the treatment of acute leukemia. Cancer Cell. 2018;33(3):495-511. https://doi.org/10.1016/j.ccell. 2018.02.002.

12. Lalic H, Dembitz V, Lukinovic-Skudar V, Banfic H, Visnjic D. 5Aminoimidazole-4-carboxamide ribonucleoside induces differentiation of acute myeloid leukemia cells. Leuk Lymphoma. 2014;55(10):2375-83. https:// doi.org/10.3109/10428194.2013.876633.

13. Dembitz V, Tomic B, Kodvanj I, Simon JA, Bedalov A, Visnjic D. The ribonucleoside AICAr induces differentiation of myeloid leukemia by activating the ATR/Chk1 kinase via pyrimidine depletion. J Biol Chem. 2019; 294(42):15257-70. https://doi.org/10.1074/jbc.RA119.009396.

14. Lewis TA, Sykes DB, Law JM, Muñoz B, Rustiguel JK, Nonato MC, et al. Development of ML390: a human DHODH inhibitor that induces differentiation in acute myeloid leukemia. ACS Med Chem Lett. 2016;7(12): 1112-7. https://doi.org/10.1021/acsmedchemlett.6b00316.

15. Wu D, Wang W, Chen W, Lian F, Lang L, Huang Y, et al. Pharmacological inhibition of dihydroorotate dehydrogenase induces apoptosis and differentiation in acute myeloid leukemia cells. Haematologica. 2018;103(9): 1472-83. https://doi.org/10.3324/haematol.2018.188185.

16. Sainas S, Pippione AC, Lupino E, Giorgis M, Circosta P, Gaidano V, et al. Targeting myeloid differentiation using potent 2-hydroxypyrazolo[1,5a]pyridine scaffold-based human dihydroorotate dehydrogenase inhibitors. J Med Chem. 2018;61(14):6034-55. https://doi.org/10.1021/acs. jmedchem.8b00373.

17. Cao L, Weetall M, Trotta C, Cintron K, Ma J, Kim MJ, et al. Targeting of hematologic malignancies with PTC299, a novel potent inhibitor of dihydroorotate dehydrogenase with favorable pharmaceutical properties. Mol Cancer Ther. 2018;18(1):3-16. https://doi.org/10.1158/1535-7163.MCT18-0863.

18. Christian S, Merz C, Evans L, Gradl S, Seidel H, Friberg A, et al. The novel dihydroorotate dehydrogenase (DHODH) inhibitor BAY 2402234 triggers differentiation and is effective in the treatment of myeloid malignancies. Leukemia. 2019;33(10):2403-15. https://doi.org/10.1038/s41375-019-0461-5.

19. Zhou J, Quah JY, Ng Y, Chooi J-Y, Toh SH-M, Lin B, et al. ASLAN003, a potent dihydroorotate dehydrogenase inhibitor for differentiation of acute myeloid leukemia. Haematologica. 2019; doi:https://doi.org/10.3324/ haematol.2019.230482

20. ClinicalTrials.gov. U.S. National Library of Medicine. 2020. https://clinicaltrials. gov/. Accessed 22 Apr 2020

21. Sasca D, Hähnel PS, Szybinski J, Khawaja K, Kriege O, Pante SV, et al. SIRT1 prevents genotoxic stress-induced p53 activation in acute myeloid leukemia. Blood. 2014;124(1):121-33. https://doi.org/10.1182/blood-2013-11538819.

22. Jones TR, Carpenter AE, Lamprecht MR, Moffat J, Silver SJ, Grenier JK, et al. Scoring diverse cellular morphologies in image-based screens with iterative feedback and machine learning. Proc Natl Acad Sci. 2009;106(6):1826-31. https://doi.org/10.1073/pnas.0808843106.

23. Yu G, Wang L-G, Han Y, He Q-Y. clusterProfiler: an R package for comparing biological themes among gene clusters. Omi A J Integr Biol. 2012;16(5):2847. https://doi.org/10.1089/omi.2011.0118.

24. The Cancer Genome Atlas Research Network. Genomic and Epigenomic landscapes of adult De novo acute myeloid Leukemia. N Engl J Med. 2013: 368(22):2059-74. https://doi.org/10.1056/NEJMoa1301689.

25. Klein H-U, Ruckert C, Kohlmann A, Bullinger L, Thiede C, Haferlach T, et al. Quantitative comparison of microarray experiments with published leukemia related gene expression signatures. BMC Bioinformatics. 2009; 10(1):422. https://doi.org/10.1186/1471-2105-10-422.

26. Tyner JW, Tognon CE, Bottomly D, Wilmot B, Kurtz SE, Savage SL, et al. Functional genomic landscape of acute myeloid leukaemia. Nature. 2018; 562(7728):526-31. https://doi.org/10.1038/s41586-018-0623-z.

27. Bagger FO, Kinalis S, Rapin N. BloodSpot: a database of healthy and malignant haematopoiesis updated with purified and single cell mRNA sequencing profiles. Nucleic Acids Res. 2019;47(D1):D881-5. https://doi.org/ 10.1093/nar/gky1076.

28. Cerami E, Gao J, Dogrusoz U, Gross BE, Sumer SO, Aksoy BA, et al. The cBio Cancer genomics portal: an open platform for exploring multidimensional Cancer genomics data: figure 1. Bönig H, editor. Cancer Discov. 2012;2(5): 401-4. https://doi.org/10.1158/2159-8290.CD-12-0095.

29. Dembitz V, Lalic $H$, Ostojic A, Vrhovac R, Banfic $H$, Visnjic D. The mechanism of synergistic effects of arsenic trioxide and rapamycin in acute myeloid leukemia cell lines lacking typical t(15;17) translocation. Int J Hematol. 2015: 102(1):12-24. https://doi.org/10.1007/s12185-015-1776-2. 
30. Visnjic D, Dembitz V, Lalic H. The role of AMPK/mTOR modulators in the therapy of acute myeloid leukemia. Curr Med Chem. 2019;26(12):2208-29. https://doi.org/10.2174/0929867325666180117105522.

31. Liu X, Chhipa RR, Pooya S, Wortman M, Yachyshin S, Chow LML, et al. Discrete mechanisms of mTOR and cell cycle regulation by AMPK agonists independent of AMPK. Proc Natl Acad Sci U S A. 2014;111(4):E435-44. https://doi.org/10.1073/pnas.1311121111.

32. Vincent EE, Coelho PP, Blagih J, Griss T, Viollet B, Jones RG. Differential effects of AMPK agonists on cell growth and metabolism. Oncogene. 2015; 34(28):3627-39. https://doi.org/10.1038/onc.2014.301.

33. Mangano DT. Effects of acadesine on myocardial infarction, stroke, and death following surgery: a meta-analysis of the 5 international randomized trials. JAMA. 1997;277(4):325-32. https://doi.org/10.1001/jama.1997. 03540280063035.

34. Narkar VA, Downes M, Yu RT, Embler E, Wang Y-X, Banayo E, et al. AMPK and PPARS; agonists are exercise mimetics. Cell. 2008;134(3):405-15. https:// doi.org/10.1016/j.cell.2008.06.051.

35. Bost F, Decoux-Poullot A-G, Tanti JF, Clavel S. Energy disruptors: rising stars in anticancer therapy? Oncogenesis. 2016;5:e188.

36. Campàs C, Santidrián AF, Domingo A, Gil J. Acadesine induces apoptosis in B cells from mantle cell lymphoma and splenic marginal zone lymphoma. Leukemia. 2005;19(2):292-4. https://doi.org/10.1038/sj.leu.2403593.

37. Santidrián AF, González-Gironès DM, Iglesias-Serret D, Coll-Mulet L, Cosialls AM, de Frias M, et al. AICAR induces apoptosis independently of AMPK and p53 through up-regulation of the BH3-only proteins BIM and NOXA in chronic lymphocytic leukemia cells. Blood. 2010;116(16):3023-32. https://doi. org/10.1182/blood-2010-05-283960.

38. Vakana E, Altman JK, Glaser H, Donato NJ, Platanias LC. Antileukemic effects of AMPK activators on BCR-ABL-expressing cells. Blood. 2011;118(24):6399402. https://doi.org/10.1182/blood-2011-01-332783.

39. Van Den Neste E, Cazin B, Janssens A, González-Barca E, Terol MJ, Levy V, et al. Acadesine for patients with relapsed/refractory chronic lymphocytic leukemia (CLL): a multicenter phase I/II study. Cancer Chemother Pharmacol. 2013;71(3):581-91. https://doi.org/10.1007/s00280-012-2033-5.

40. Huang M, Wang Y, Collins M, Mitchell BS, Graves LM. A77 1726 induces differentiation of human myeloid leukemia $\mathrm{K} 562$ cells by depletion of intracellular CTP pools. Mol Pharmacol. 2002;62(3):463-72. https://doi.org/10. $1124 / \mathrm{mol} .62 .3 .463$

41. Sykes DB. The emergence of dihydroorotate dehydrogenase (DHODH) as a therapeutic target in acute myeloid leukemia. Expert Opin Ther Targets. 2018;22(11):893-8. https://doi.org/10.1080/14728222.2018.1536748.

\section{Publisher's Note}

Springer Nature remains neutral with regard to jurisdictional claims in published maps and institutional affiliations.

Ready to submit your research? Choose BMC and benefit from:

- fast, convenient online submission

- thorough peer review by experienced researchers in your field

- rapid publication on acceptance

- support for research data, including large and complex data types

- gold Open Access which fosters wider collaboration and increased citations

- maximum visibility for your research: over $100 \mathrm{M}$ website views per year

At $\mathrm{BMC}$, research is always in progress.

Learn more biomedcentral.com/submissions 